\title{
Quadratic effects in the nonlinear magneto-optical response of perovskite manganites studied with magnetization-induced second harmonic generation
}

\author{
E. D. Mishina, ${ }^{1,2,3}$ A. I. Morosov, ${ }^{3}$ A. V. Mishina, ${ }^{3}$ V. Moshnyaga, ${ }^{2}$ L. Sudheendra, ${ }^{2}$ K. Samwer, ${ }^{2}$ and Th. Rasing ${ }^{1}$ \\ ${ }^{1}$ Institute for Molecules and Materials, Radboud University Nijmegen, Toernooiveld 1, 6525ED Nijmegen, The Netherlands \\ ${ }^{2}$ I.Physikalisches Institut, Universität Göttingen, Göttingen, Germany \\ ${ }^{3}$ Moscow State Institute of Radioengineering, Electronics and Automation, prosp. Vernadskogo 78, 119454 Moscow, Russia
}

(Received 30 August 2006; published 1 February 2007)

\begin{abstract}
The crystallographic and magnetic symmetry of centrosymmetric perovskite manganite epitaxial films is studied by magnetization-induced optical second harmonic generation (MSHG). It is shown that not only linear but also quadratic terms in the magnetization affect the nonlinear-optical response, the latter being even dominant for special geometries. In that case MSHG allows to simultaneously probe the crystallographic structure, the net magnetization (zero in demagnetized material), and magnetization squared (nonzero in demagnetized material).
\end{abstract}

DOI: 10.1103/PhysRevB.75.064401

PACS number(s): 75.47.Lx, 78.20.Ls

\section{INTRODUCTION}

Perovskite manganites like $\mathrm{La}_{1-x} \mathrm{Ca}_{x} \mathrm{MnO}_{3}$ (LCMO) represent a class of colossal magnetoresistive (CMR) materials, which are at the focus of fundamental and applied research. These strongly correlated systems possess unusual coupled magnetic, transport and optical properties, which have potential for different applications like bolometric detectors, ${ }^{1}$ spinvalve structures, ${ }^{2}$ or magnetic field sensors. ${ }^{3}$ The interplay between spin, charge, lattice, and orbital degrees of freedom may lead to very complex and spatially inhomogeneous structures with an enormous diversity of phases and properties to explore, increasing the potential for novel behavior. ${ }^{4}$

Magneto-optical effects linear in magnetization are widely used for investigation of thin film magnetism. In particular, the linear magneto-optical Kerr effect (MOKE) is applied to study magnetic anisotropy and magnetization reversal. It was shown ${ }^{5,6}$ that those measurements can be significantly affected by quadratic contributions in the magnetization, analogously to the Voigt or Cotton-Mouton effect. Since higher-order optical tensors are involved, new effects were found via these terms. For example, for a cubic crystal, which is normally isotropic for linear optics, anisotropy in reflectivity was found as a result of higher order contributions. ${ }^{5,6}$

Nonlinear magneto-optical effects [in particular, magnetization-induced second harmonic generation (MSHG)] are intrinsically described by higher order optical tensors in comparison with MOKE. ${ }^{7}$ This gives several advantages: most of all, because of its surface/interface sensitivity, the nonlinear magneto-optical response provides information on the magnetization of surfaces and buried interfaces separately from the bulk. ${ }^{8}$ Due to different selection rules and higher-order optical tensors, also new magneto-optical effects may arise in MSHG, that allow the magneto-optical observation of antiferromagnetic domains ${ }^{9}$ and of complicated domain patterns in strained films of, for example, yttrium-iron-garnet. ${ }^{10}$ As MSHG is sensitive to the arrangement of both charge and spin, their contributions allow to probe local or hidden phase transitions, interacting magnetized and polarized sublattices and domain walls.
In this paper we report the results of a nonlinear-optical study of the $A$-site ordered LCMO thin film. From a symmetry analysis, based on the nature of the nonlinear optical sources, and experimental temperature and magnetic field dependences of the SHG intensity and magnetization below magnetic phase transition we found along usual magnetization- and crystallographic-induced SHG components, an additional strong contribution to crystallographic SHG, which is proportional to the square of local magnetization. Moreover, the magnitude of this crystallographic correction is found to be unexpectedly high.

\section{EXPERIMENT AND RESULTS}

Epitaxial $\mathrm{La}_{0.75} \mathrm{Ca}_{0.25} \mathrm{MnO}_{3}$ (LCMO) film was prepared by a metalorganic aerosol deposition (MAD) technique ${ }^{11}$ on $\mathrm{MgO}(100)$ substrates (crystal $\mathrm{GmbH}$, miscut less than 0.3). The thickness of the film as measured by small angle $\mathrm{x}$-ray scattering was $90 \mathrm{~nm}$; and the Curie temperature, $T_{\mathrm{C}}$ $=263 \mathrm{~K}$. X-ray diffraction analysis shows "cube-on-cube" epitaxy of LCMO on $\mathrm{MgO}(100)$ and indicates a stress-free state of the film: the out-of plane lattice parameter, $c$ $=0.3877 \mathrm{~nm}$, is very close to the bulk value. ${ }^{12}$ Remarkably, LCMO film shows a unique perovskite superstructure due to CE-type ordering of $\mathrm{La}$ and $\mathrm{Ca}$, accompanied by the formation of a pseudocubic or rhombohedral $(R-3 c)(3-m)$ crystalline structure, observed by transmission electron microscopy and electron diffraction analysis. ${ }^{12}$ The obtained structure is different from the orthorhombic one, which is generally observed for LCMO bulk crystals and thin films. ${ }^{13}$

Optical SHG was excited by the output of a Ti:sapphire laser $\left(\lambda_{\omega}=780 \mathrm{~nm}, \Delta t_{\text {pulse }}=100 \mathrm{fs}\right.$, repetition rate $82 \mathrm{MHz}$, pulse power density up to $5 \times 10^{9} \mathrm{~W} / \mathrm{cm}^{2}$ ). Polarization of the fundamental wave was controlled by a Berek compensator and of the SH waves by a Glan prism (in the following, $p-, s$-, and $m$ - polarization of the electric field vector indicate orientations parallel, perpendicular, and at $45^{\circ}$ to the plane of incidence, respectively). For all SHG measurements except for the azimuthal dependences, the transmission geometry was used at an angle of incidence of $30^{\circ}$. The samples were oriented with the [001] axis of the substrate perpendicular to 


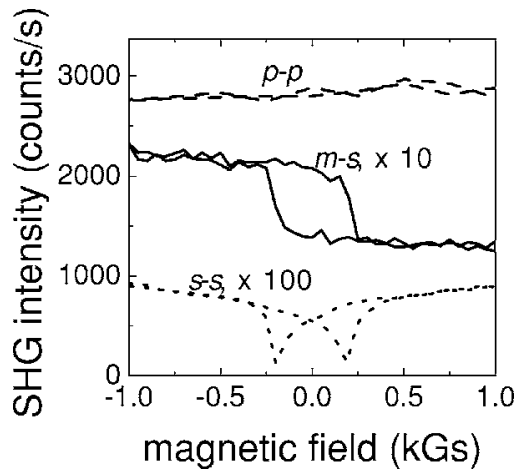

FIG. 1. Hysteresis loops of SHG intensity in LCMO for different polarization combinations measured at $10 \mathrm{~K}$.

the plane of incidence. Azimuthal dependences of SHG were measured in the reflection geometry at an angle of incidence of $45^{\circ}$ the samples were rotated around their normal. The SHG signal was discriminated spectroscopically by means of appropriate color and band-pass filters and then detected by a photon counting system. The temperature dependences of SHG were measured for $T=10-300 \mathrm{~K}$ on heating by using a He cryostat. All temperature dependent measurements were performed during heating. Both ZFC (zero field cooling) and FC (cooling under a magnetic field of $H= \pm 1 \mathrm{kGs}$ ) samples were used for these temperature scans. The magnetic field was aligned in the film plane (longitudinal Kerr geometry). Optical constants ( $n$ and $k$ ) were measured in the temperature range of $100-300 \mathrm{~K}$ by spectroscopic ellipsometry (J. A. Woollam Co., Inc.). They show linear temperature dependences above $T_{\mathrm{C}}$ and critical behavior in the vicinity of the phase transition.

The most intriguing result is that the magnetic field dependence of MSHG (Fig. 1) changes essentially with the polarization combinations of fundamental and SHG waves, whereas the temperature dependence of the MSHG (Fig. 2) does not. The most straightforward results were observed for the $m-s$ polarization combination: (i) an MSHG loop (Fig. 1) that is symmetric with regards to the center of the loop

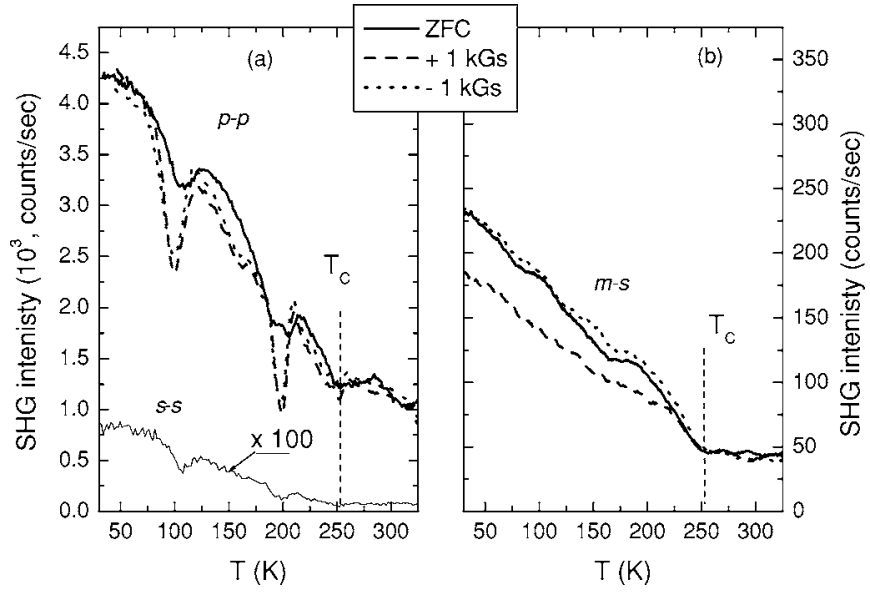

FIG. 2. Temperature dependences of SHG intensity in LCMO for $p-p$ and $s-s$ (a) and $m-s$ (b) polarization combinations for zero field cooled sample and also with magnetic field applied. ("centro-symmetric loop"), very similar to the magnetization curve or MOKE loop; ${ }^{14}$ and (ii) temperature dependences [Fig. 2(b)] for positively and negatively oriented magnetic fields that are very similar and differ from each other only by the absolute value of the signal in the ferromagnetic state, $T<T_{\mathrm{C}}$. Remarkably, the ZFC data do not lie in the middle between those for oppositely magnetized samples, but is almost coincident with one of them.

For the $s-s$ polarization combination the MSHG data show a hysteresis behavior around zero field that is distinctly different from a normal magnetization loop but instead shows a symmetric minimum on either side ("axissymmetric loop"). The corresponding temperature dependences [Fig. 2(a)] for oppositely directed magnetic fields are coincident within the error bar.

The $p-p$ polarization combination shows no magnetic field dependence at all (Fig. 1) and consequently the temperature [Fig. 2(a)] dependences of the MSHG signals for $H=0$ or $H= \pm 1 \mathrm{kG}$ practically coincide except near some sharp dips.

In addition to the temperature and magnetic field dependences, standard azimuthal $\left(I^{2 \omega}(\Psi)\right)$ and polarization $\left(I^{2 \omega}(\varphi)\right)$ dependences of the MSHG intensity were measured, which allow one to perform a complete analysis of the MSHG signal.

The MSHG intensity dependencies on azimuthal angle $I^{2 \omega}(\Psi)$, measured at room temperature in the reflection geometry, possesses slightly deformed eightfold symmetry in both $p-s$ and $s-s$ polarization combinations (Fig. 3).

For $I^{2 \omega}(\varphi)$ (Fig. 4), the output polarization was kept constant, either $p$ or $s$, and $\varphi=0$ corresponds to $s$ input polarization (see inset in Fig. 4). For these measurements the sample was kept in a cryostat at fixed azimuthal angle $\Psi=0$, which corresponded to the (001) axis of the substrate being parallel to the electric field vector of $s$-polarized light (oriented vertically).

\section{DISCUSSION}

\section{A. Theoretical description of magnetization-induced SHG}

A fundamental electromagnetic wave with wave vector $\vec{k}$, electric field strength $\vec{E}(\omega)$, and magnetic field strength $\vec{H}(\omega)$ incident on a material induces an electric polarization $\vec{P}$ and magnetization $\vec{M}$. To describe the response of the medium these excitations are expanded in terms of powers of $\vec{E}(\omega)$ and also in terms of multipole momenta. ${ }^{15}$ In order to describe the medium response at the SHG frequency $2 \omega$, the standard wave equation should be solved with the following source term:

$$
\vec{S}(2 \omega)=\mu_{0} \frac{\partial^{2} \vec{P}}{\partial t^{2}}+\mu_{0}\left(\vec{\nabla} \times \frac{\partial \vec{M}}{\partial t}\right)-\mu_{0}\left(\vec{\nabla} \frac{\partial^{2} \hat{Q}}{\partial t^{2}}\right),
$$

where $\vec{P}, \vec{\nabla} \hat{Q}$, and $\vec{M}$ are induced electric dipole polarization, magnetization, and electric quadrupole polarization, respectively. 


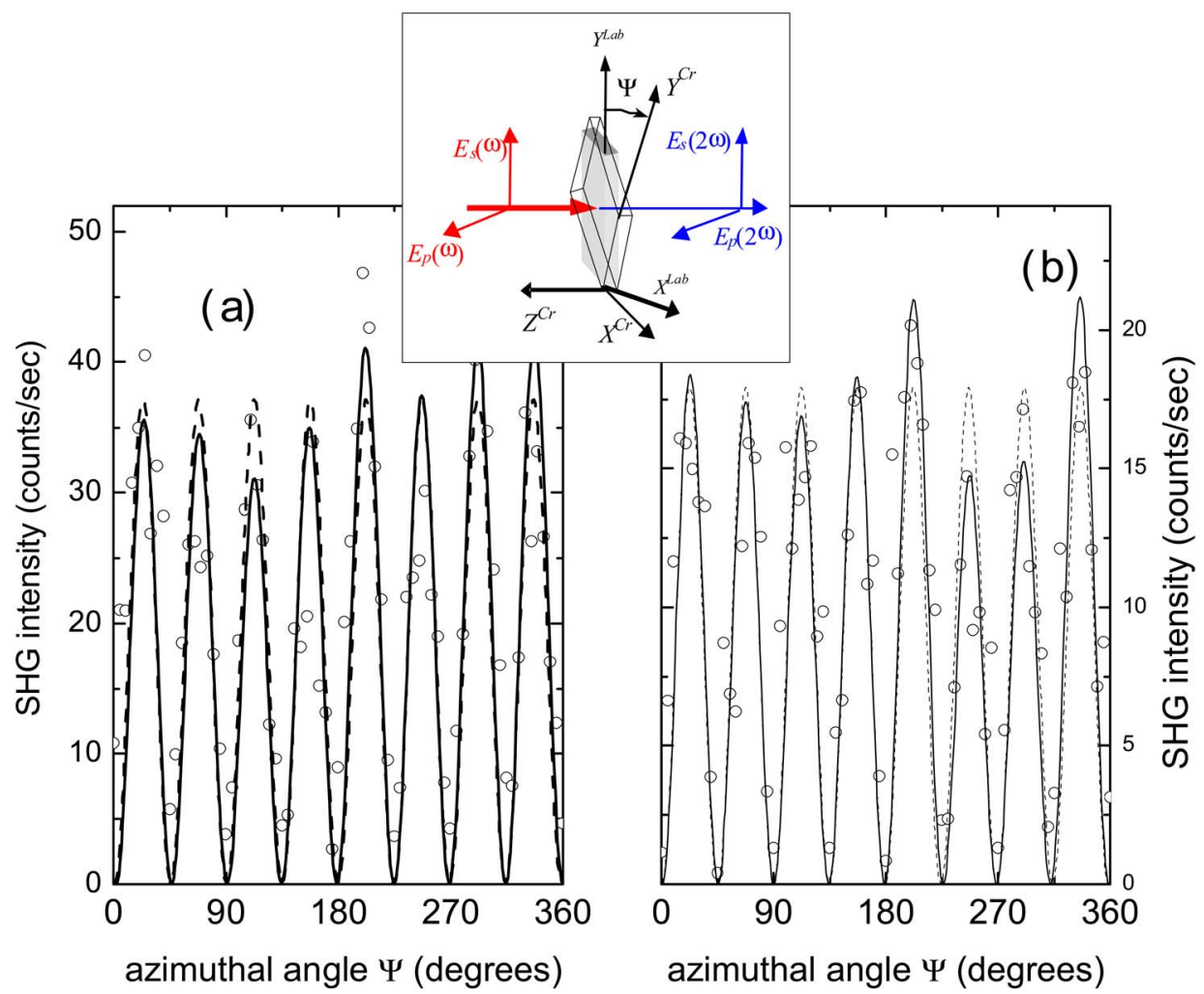

FIG. 3. (Color online) Rotational anisotropic dependences of SHG intensity in LCMO at room temperature $p-s($ a) and $s-s$ (b) polarization combinations: experiment (circles), fits to data using orthorhombic (solid line) and cubic (dashed line) symmetry. Inset-geometry of experiment: $X^{C r}, Y^{C r}, Z^{C r}$ crystallographic quasicubic frame, $X^{L a b}, Y^{L a b}, Z^{L a b}$ laboratory frame. Azimuthal angle $\Psi$ is measured between $X^{C r}$ and $X^{L a b}$.
In centrosymmetric media in the absence of a net magnetization three types of nonlinear sources may contribute to the SHG signal: ${ }^{15}$

(i) an electric polarization of quadrupole type (Q), which after taking the gradient of $\hat{Q}$ yields

$$
P_{i}^{Q}(2 \omega) \propto i \chi_{0 i j k l}^{Q} E_{j}(\omega) k_{k} E_{l}(\omega),
$$

(ii) a magnetization of dipole type:

$$
M_{i}^{M D M}(2 \omega) \propto \chi_{0 i j k}^{M D M} E_{j}(\omega) E_{k}(\omega),
$$

(iii) an electric polarization of magnetic-dipole type:

$$
P_{i}^{M D P}(2 \omega) \propto \chi_{0 i j k}^{M D P} E_{j}(\omega) H_{k}(\omega),
$$

where the $\hat{\chi}$ are nonlinear susceptibility tensors of different nature, which are generally determined by spatial and timereversal symmetry. ${ }^{16}$ The magnetic-dipole tensors $\hat{\chi}_{0}^{M D M}$ and $\hat{\chi}_{0}^{M D P}$ have identical nonzero tensor components but their values are different, because of the absence of the $j-k$ exchange symmetry for $\hat{\chi}_{0}^{M D P}$. Terms (2)-(4) are of the same order of magnitude. In the following they will be called crystallographic, because they do not depend on net magnetization. However, in some cases they may be separated either by spectroscopic measurements (as in Ref. 15), or by azimuthal anisotropic measurements (as in this work).

In the presence of a local magnetization $\mu_{i}$, the magnetization-induced contributions (MI) are added to the crystallographic contributions; the local MI contributions [local polarization $p_{i}^{P M I, l o c}(2 \omega)$ and local magnetization $\left.m_{i}^{M M I, l o c}(2 \omega)\right]$ are expressed in terms of local magnetization:

$$
p_{i}^{P M I, l o c}(2 \omega) \propto \chi_{i j k l}^{P M I} \mu_{j} E_{k}(\omega) H_{l}(\omega),
$$

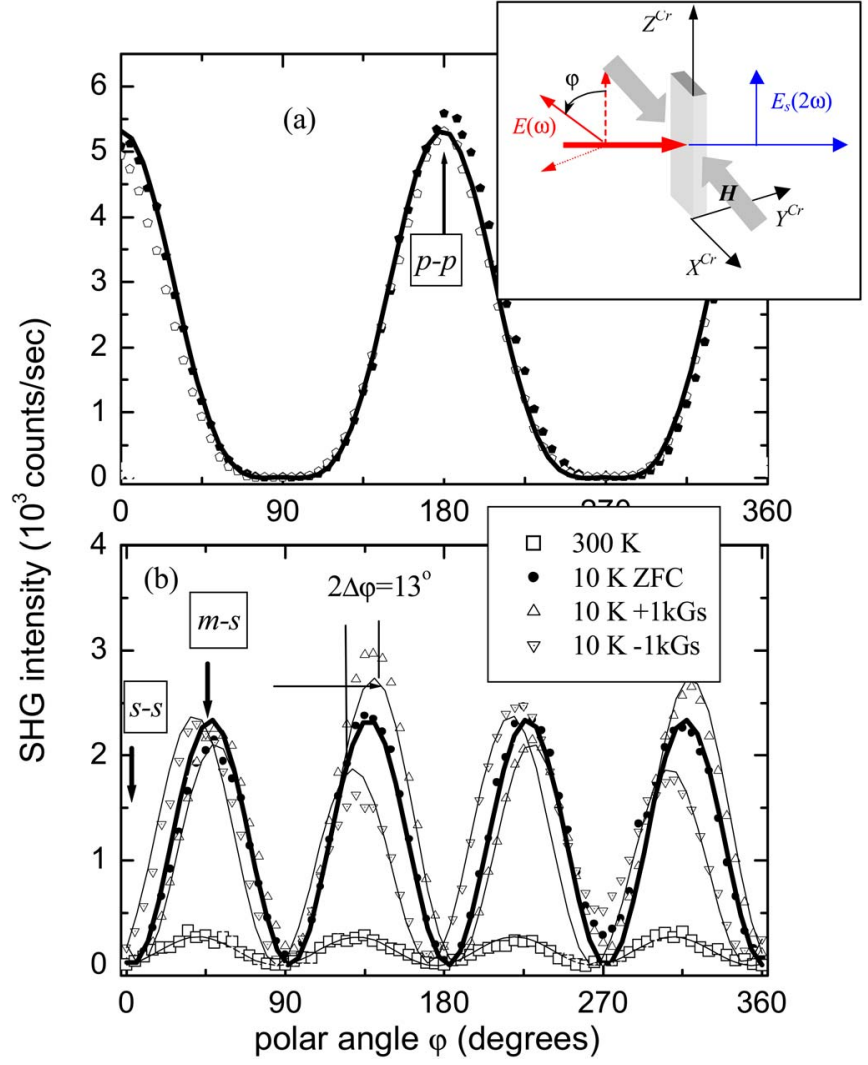

FIG. 4. (Color online) Polarization dependences of SHG intensity $I^{2 \omega}(\varphi)$ in LCMO films at room temperature and $10 \mathrm{~K}$ for $p$-out (a) and $s$-out (b) SHG polarization. In ferromagnetic phase magnetic field was applied parallel to crystallographic $a$ axis. Linesfits to data within the model described in the text. Inset—geometry of experiment. 


$$
m_{i}^{M M I, l o c}(2 \omega) \propto \chi_{i j k l}^{M M I} \mu_{j} E_{k}(\omega) E_{l}(\omega) .
$$

Note that (5) and (6) represent the first terms in a Tailor expansion in the magnetization; therefore the tensors $\hat{\chi}^{P(M) M I}$ are taken at $\mu_{i}=0$, i.e., in the high temperature (paramagnetic) phase. They are generally of the same order of magnitude and relativistically small in comparison with electricdipole term of the type

$$
p_{i}^{M I} \propto \chi_{i j k l}^{M I} \mu_{j} E_{k} E_{l}
$$

because both vectors $\vec{H}$ in (5) and $\vec{m}$ in (6) are of $v / c$ smaller in comparison with $\vec{E}$ and $\vec{p}$, respectively ( $v$ is characteristic velocity of electron in the system). However in the case of space-inversion symmetry the electric-dipole term (7) is absent and terms (5) and (6) are the only contributing to MSHG.

For a multidomain sample the polarization (5) and magnetization (6) should be averaged over the probing light spot

$$
\begin{gathered}
P_{i}^{P M I}(2 \omega) \propto \chi_{i j k l}^{P M I} M_{j} E_{k}(\omega) H_{l}(\omega), \\
M_{i}^{M M I}(2 \omega) \propto \chi_{i j k l}^{M M I} M_{j} E_{k}(\omega) E_{l}(\omega),
\end{gathered}
$$

where $M_{j}=\Sigma\left(\Delta F^{+} \mu_{j}^{+}+\Delta F^{-} \mu_{j}^{-}\right)$is the macroscopic (net) magnetization, $\Delta F^{+}$and $\Delta F^{-}$are the fractions of domains oppositely oriented along the direction $j$. Symmetry, including time-inversion, determines the type of susceptibility tensors $\hat{\chi}$ in $(2)-(6)$.

In the absence of absorption the quadrupole tensor $\chi_{i j k l}^{Q}$ is polar and independent on time-reversion. ${ }^{16}$ The magneticdipole tensors $\chi_{i j k}^{M D M}$ and $\chi_{i j k}^{M D P}$ are axial and change sign with time reversal. The $\chi_{i j k}^{M I}$ tensors in (7) and (8) are of the same type as the quadrupole tensor, i.e., polar and independent on time reversion. In the presence of absorption all terms have both real and imaginary parts providing interference between all of them.

For any centrosymmetric medium, the surface dipole-type polarization due to the symmetry breaking surfaces is generally of the same order of magnitude as the bulk (quadrupole in this case) polarization. It can be written as

$$
P_{i}^{S}(2 \omega) \propto \chi_{i j k}^{S} E_{j}(\omega) E_{k}(\omega) .
$$

Nonzero components of the $\hat{\chi}^{S}$ tensor are determined by the $4 \mathrm{~mm}$ surface symmetry.

Generally, the anisotropic and polarization dependence of the SHG signal from a single crystal can be expressed as a truncated Fourier expansion: ${ }^{17}$

$$
\begin{gathered}
E^{2 \omega}(\Psi)=\left(f_{0}+\sum_{n=1}^{4}\left(c_{n} \cos (n \Psi)+s_{n} \sin (n \Psi)\right)\right) E^{2}(\omega) \\
E^{2 \omega}(\varphi)=\left(F_{0}+C_{2} \cos 2 \varphi+S_{2} \sin 2 \varphi\right) E^{2}(\omega)
\end{gathered}
$$

In Eqs. (11) and (12) the Fourier coefficients $f_{0}, c_{i}, s_{i}$, and $F_{0}, C_{i}, S_{i}$ are determined by the sum of the products of the Fresnel factors depending on the polarization angle $\varphi$ and on the azimuthal angle $\Psi$ and appropriate tensor components. ${ }^{17}$
The symmetry of the hysteresis loop may show which components are responsible for the SHG field. A "centrosymmetric loop" corresponds to a dependence, in which both crystallographic and magnetization induced terms are present: $I_{m-s}^{2 \omega}(M) \propto(\text { const }+M)^{2}$. An "axisymmetric loop" means that the crystallographic contribution is absent and the signal is purely magnetic $I_{s-s}^{2 \omega}(M) \propto\left(P_{s-s}^{M I}\right)^{2} \propto M^{2}$. The nonzero MSHG intensity in the minima arises due to the complex values of the nonlinear susceptibility. Very small dependence on magnetization of $p$ - $p$ polarized SHG means that the crystallographic contribution is dominant for this case.

Analysis of the azimuthal and polarization dependences of the SHG intensity allows to separate the crystallographic and magnetization induced terms. Analysis was performed (see Appendix A) for rhombohedral and cubic crystals which show that according to the SHG measurements the deviation of the bulk crystal symmetry from the cubic is very small. Based on this conclusion in the following the obtained experimental temperature dependences were analyzed for the case of cubic symmetry.

From symmetry analysis it also follows that under the experimental conditions MDM and MDP terms do not contribute to SHG in most polarization combinations (see Appendix A).

It is necessary to carefully analyze possible surface contributions. For cubic bulk symmetry, the surface point group symmetry is $4 \mathrm{~mm}$, which gives in $s-s$ and $p-s$ polarization combinations $E_{2 \omega}^{S}(\Psi) \equiv 0$. For rhombohedral bulk symmetry, the surface point group symmetry is $2 \mathrm{~mm}$, which gives $E_{2 \omega}^{S}(\Psi) \equiv 0$ in $s-S$ polarization combinations. Therefore in the following the obtained experimental temperature dependences were analyzed with the bulk contribution only taken into account.

\section{B. Temperature dependences}

From the polarization dependences at different temperatures (Fig. 4) it follows that both the magnetization induced and the crystallographic contributions are temperature dependent. For the MI contribution, the temperature dependence arises from the temperature dependence of the net magnetization $M(T)$. For the crystallographic contribution the nature of the temperature dependence has to be clarified.

Additional information can be obtained from an analysis of the MSHG temperature dependences for different polarization combinations. Generally for our samples, decreasing the temperature down from $T_{\mathrm{C}}$ results in a significant increase of the MSHG intensity for all polarization combinations (Fig. 2). Qualitatively, all dependences do not show a significant difference between $\mathrm{ZFC}$ and FC. The continuous growth of intensity with decreasing temperature is interrupted by quite deep equidistant dips. These are the result of multiple reflections and interference of light in the film/ substrate system, due to the change of optical path with temperature. This is analogous to the effect observed in Ref. 18, where multiple reflection of fundamental and SHG waves at all interfaces gives corrections (additional fine interference patterns) for the SHG dependence on a the angle of incidence, so-called Maker-fringes. The varying depth of the 
dips is due to different positions of the laser spot on the film surface.

For the $m$ - $s$ polarization combination, a clear difference in temperature dependence (up to 20-30 \% at 40 K, see Figs. 1 and 2) is observed for the two opposite directions of magnetic field. Interestingly, $I^{2 \omega}(T)$ for ZFC conditions is almost coincident with the dependence for MSHG for negatively oriented field. The difference in temperature dependences for positively and negatively oriented magnetic field agrees also with the SHG hysteresis loop (significantly different SHG intensity for $\pm 1 \mathrm{kGs}$, Fig. 1).

For the $p-p$ polarization combination, the temperature dependences for positively and negatively oriented magnetic field coincide within the error bar (Fig. 2). This is in agreement with the absence of hysteresis (Fig. 1) and also with the complete coincidence of the polarization dependences for $p$-out SHG intensity for the two directions of magnetic field (not shown here). The ZFC temperature dependence is different from the field induced ones only in the areas of the interference dips, and is very similar elsewhere.

For the $s-s$ polarization combination, the MSHG intensity temperature dependences are in agreement with the $s$-s hysteresis loop.

The different shapes of hysteresis loops for different polarization combinations follow directly from the analysis of the various contributing components.

The shape of the hysteresis loop for the $m-s$ polarization combination is determined by the interference of $Q, M D P$ and $M I$ contributions: $I^{2 \omega} \propto(Q+M D P+M I(M))^{2}$, which gives a centrosymmetric shape for $M I(M)<Q, M D P$. Interference between these contributions explains also the asymmetry of the temperature dependence with respect to the magnetic field direction. For the $s-s$ polarization combination $I^{2 \omega} \propto\left(P^{M I}\right)^{2} \propto M^{2}$, which yields a loop symmetric with regards to the vertical axis and a coincidence of temperature dependences for opposite directions of magnetic field. For the $p$ - $p$ polarization combination generally both MI and crystallographic terms should contribute to the MSHG. However the absence of magnetic field dependence means that $M I \ll Q, M D P$ and hence $I^{2 \omega} \propto\left(P^{Q, M D P}\right)^{2}$.

Thus, the observed temperature dependences and hysteresis loops of the SHG intensity allow us to conclude: the crystallographic contribution depends strongly on temperature (for the $p-p$ polarization combination the total signal mostly comes from the crystallographic contributions, for the $s-s$ polarization combination, the ZFC temperature dependence is also due to the crystallographic contribution); comparison of crystallographic and magnetization-induced contributions (for $p-p$ and $s-s$ polarization combinations) shows the similarity of their critical behavior: both increase significantly with decreasing temperature below $T_{\mathrm{C}}$.

There are three possible reasons for the observed temperature dependence of the MI contribution (including its critical behavior): (i) critical behavior of the nonlinear polarization via magnetization $M(T)$ [and MSHG through Eqs. (7) and (8)]; (ii) critical behavior of the film linear optical constants via magnetization $M(T)$ (and MSHG through the Fresnel factors), (iii) noncritical temperature dependences of the film and the substrate optical constants and the substrate thickness.
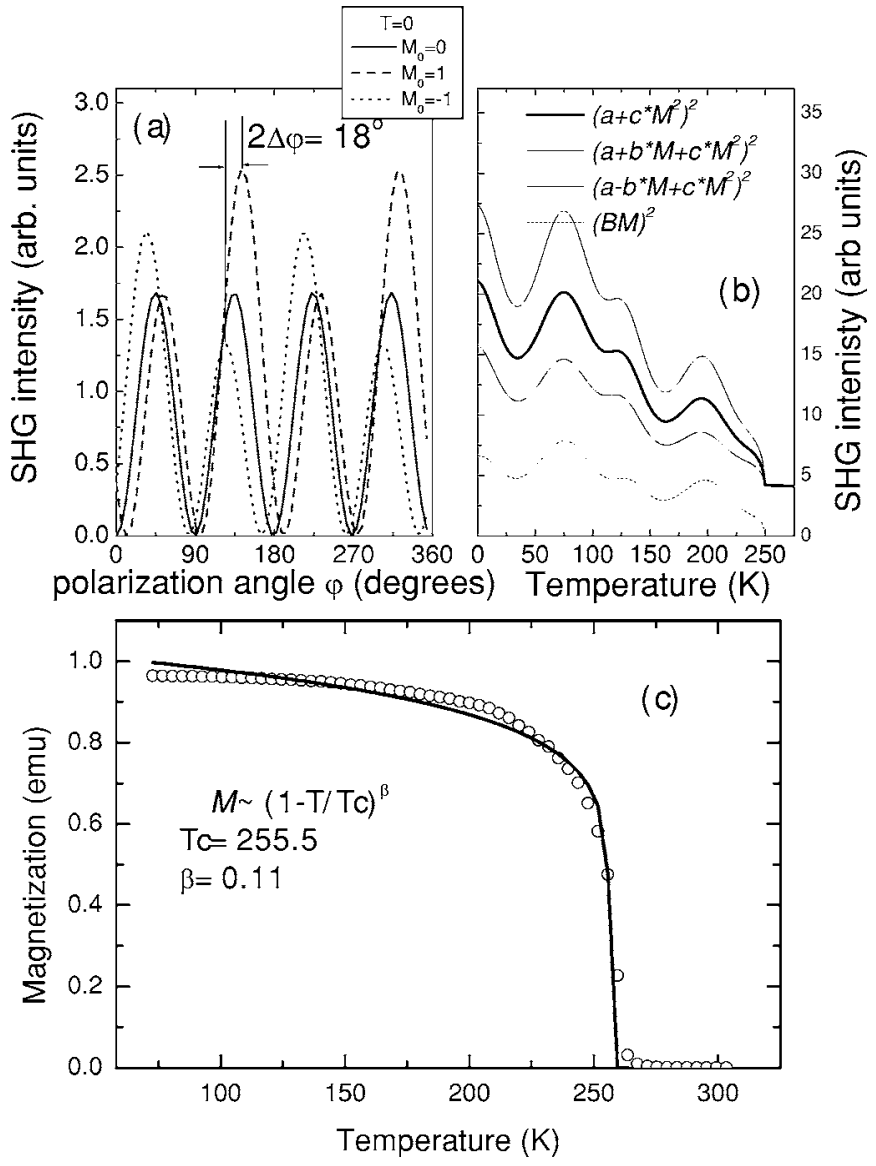

FIG. 5. Model calculations of polarization (a) and temperature (b) dependences of SHG intensity in cubic approximation. Azimuthal angle $\Psi=0$. For polarization dependence $T=0$, SHG is $s$ polarized. For temperature dependence $s-s$ and $m-s$ polarization combination is considered. Magnetization is given in arbitrary units. (c) Experimental (circles) and calculated (line) temperature dependences of magnetization.

In more details:

(i) The temperature dependence of the magnetization as measured by SQUID magnetometer is shown in Fig. 5(c). The type of magnetic phase transition for optimally doped $(0.2<x<0.4) \mathrm{La}_{1-x} \mathrm{Ca}_{x} \mathrm{MnO}_{3}$ is known to be of the first order and becomes the second order phase transition for $x$ $>0.4 .^{19,20}$ It is quite difficult sometimes to determine the order of a transition from experimental results; attempts to describe such phase transitions by scaling behavior ${ }^{21}$ resulted in anomalously low values for the critical exponent ( $\beta$ $=0.13$ ) which may be an indication that it is really a firstorder PT. For our sample the best fit of the temperature dependence of the magnetization data to a power law $M=\tau^{\beta}$ gave a critical exponent $\beta=0.11$ [Fig. 5(c), line]. This also indicates a first order phase transition. From a theoretical point of view it is quite important whether $M$ displays a discontinuous jump or is described by a power law. However, for practical purposes of fitting the MSHG data almost no difference appears between substituting to $I^{S H G}(T)$ of $M(T)$ experimental data numerically or using the analytical function $M=\tau^{\beta}$. 
(ii) From ellipsometric data we obtained sharp changes in the refractive index and absorption coefficient in the vicinity of $T_{\mathrm{C}}$ for both the fundamental and SHG wavelengths.

(iii) The temperature dependences of SHG reveal several dip features below $T_{\mathrm{C}}$. Simulations show that these dips mainly arise due to the thermal expansion of the $\mathrm{MgO}$ substrate. Concluding from (i)-(iii): For the MI contribution, which is linear in the net magnetization, the main increase of the MSHG intensity below $T_{\mathrm{C}}$ arises from (i) and (ii) via the $M(T)$ dependence, the observed dips in the $I^{S H G}(T)$ dependence are Maker-like fringes due to the thermal expansion of the substrate.

For the crystallographic contribution only (ii) and (iii) are relevant, but they are not able to explain the strong increase of $I^{S H G}(T)$ below $T_{\mathrm{C}}$, particularly for demagnetized sample.

We suggest here an alternative mechanism that may give rise to the observed temperature dependence of the MSHG, namely a magnetization-induced correction to the crystallographic contributions. The quadrupole contribution can be described as

$$
\chi_{i j k l}^{Q}(\mu)=\chi_{i j k l}^{Q}(0)+\chi_{i j k l m n}^{Q} \mu_{m} \mu_{n} .
$$

Here, the linear term in $\mu$ vanishes due to the symmetry rules $\left(\chi_{i j k l m}^{Q}=0\right.$ for $\left.3-m\right)$. Since these corrections are quadratic in local magnetization $\mu$, no hysteresis can be generated by this term, in agreement with experiment ( $p$ - $p$ polarization, see Fig. 1) and the assumption that the temperature dependence of the local magnetization $\mu(T)$ is similar to that of the net magnetization. Analogous corrections should be added to terms (3) and (4) as well and appropriate contributions will be referred to as "corrected crystallographic" contributions.

Using this, the polarization $I^{2 \omega}(\varphi)$ and temperature $I^{2 \omega}(T)$ dependences of the MSHG intensity were simulated [Figs. 5(a) and 5(b)]. Qualitatively they strongly resemble the experimental dependences $I^{2 \omega}(\varphi)$ and $I^{2 \omega}(T)$ shown in Figs. 2(b) and 4(b). The calculated polarization dependences for the $s$-out MSHG show the magnetization-induced shift of about $18^{\circ}$ (experimental value is $13^{\circ}$ ). Thus, excluding oscillations due to interference, the MSHG temperature dependences are the result of a strong dependence of the nonlinear optical susceptibility of LCMO on the magnetization (local and net).

Based on the symmetry analysis for the experimental polarization configuration we can neglect MDM contribution (3). Some arguments can be advanced in favor of quadrupole contribution (2) against MDP contribution (4).

A reason for a high value of quadrupole contribution may be a cation order induced charge/orbital ordering $(\mathrm{COO})$ and correspondent static electric quadrupole momentum arising due to the existence of long range $A$-site ordering of CE-type in LCMO film. ${ }^{12}$ It is known ${ }^{22}$ that doubly degenerated $e_{g}$ orbitals of $\mathrm{Mn}$ ions correspond to a quadrupolar distribution of the electronic density: elongated electron ellipsoid for an electron occupying $\left(3 z^{2}-r^{2}\right)$ orbital, and the flattened (compressed) ellipsoid for the $\left(x^{2}-y^{2}\right)$ orbital. The (La,Ca) ordered superstructure results in a stripe-like distribution of lattice strain with alternating stripes of compressive- and tensile-like lattice strain. ${ }^{12}$ Providing a stress compensational mechanism and, thus, electronically homogenous ground state for the mesoscopic scale, i.e., much larger than superlattice cell, $a_{\mathrm{S}}=4 a_{\mathrm{Per}} \sim 1.55 \mathrm{~nm}$, the lattice strain stripes on the microscopic scale lead to the formation of charge/orbital stripes in close agreement with quadrupolar elastic interaction proposed in. ${ }^{22}$ Very recently these stripe features were visualized by high resolution scanning tunneling microscopy (STM) and scanning tunneling spectroscopy (STS) measurements. ${ }^{23}$ Moreover the appearance of spontaneous magnetization for $T<T_{\mathrm{C}}$ and observed change of the alignment of $\mathrm{COO}$ stripes from the diagonal for $T>T_{\mathrm{C}}$ to that parallel to $(\mathrm{Mn}-\mathrm{O}-\mathrm{Mn})$ bonds $\left(T<T_{\mathrm{C}}\right)$ are both intimately coupled to the change of the sign of quadrupolar elastic interaction, quantified by the value $d=c_{11}-c_{12}-2 c_{44}$ (Ref. 22) $\left(c_{\mathrm{ij}}\right.$ are elastic moduli of the crystal). For nonlinear optics this means a strong quadrupolar unharmonicity of the oscillator, which may result in a strong increase of the quadrupole term across the phase transition. However, in order to confirm the large value of the quadrupole susceptibility, the firstprinciple calculations are necessary in the way it was recently done for $\mathrm{NiO}^{24}$

The high value of the quadratic in magnetization term (which is the second term in a Taylor expansion and therefore should be much smaller than previous terms) is quite intriguing. It can be explained in terms of quantum mechanical perturbation theory. In the absence of relativistic spinorbital and dipole-dipole interaction in the paramagnetic phase, spin and coordinate parts of the wave function are independent. Then, the electron dispersion relation is twice degenerate in the spin projection (Kramer's degeneration). If ferromagnetic ordering occurs, the exchange field $\vec{H}_{e}$ influences the spins: $\vec{H}_{e} \propto \vec{\mu}$.

Since the appropriate perturbation operator $\hat{V}$ in Hamiltonian is proportional to an electron spin projection $\hat{S}_{z}$ and contribution of electrons of opposite signs into linear and nonlinear susceptibilities are additive and opposite due to Kramer's degeneration, linear on perturbation operator (and hence on $\hat{S}_{z}$ ) corrections to susceptibility equal to zero (for details see Appendix B).

Quadratic on electric spin correction to susceptibility can be obtained in two ways:

First order on $\hat{V}$ and first order on spin-orbital interaction operator $\hat{H}$.

Second order on $\hat{V}$.

In the first case the linear in magnetization contribution to nonlinear susceptibility $\hat{\chi}_{i j k}$ contains relativistically small parameter $(v / c)^{2}$ ( $v$ is electron velocity). In the second case one obtains quadratic in magnetization contribution, which does not contain relativistic small parameter. Since spin and coordinate spaces are not connected, this contribution does not depend on magnetization direction and should be proportional to $\vec{\mu}^{2}$. Contribution containing $\mu_{i} \mu_{j}(i \neq j)$ could appear only as relativistically small. Far form phase transition in ferromagnetic phase, where expansion in series over magnetization may be not valid, pure exchange contribution (second order on $\hat{V}$ ) should become dominant 


\section{CONCLUSIONS}

We studied magnetization induced SHG (MSHG) in epitaxial $A$-site ordered LCMO film. In agreement with structural studies, in nonlinear optics the films possess themselves as quasicubic, i.e., more symmetrical in comparison with the bulk crystal and the films fabricated by different techniques.

The observed MSHG is essentially affected by the $M^{2}$ contribution to the quadrupole susceptibility tensor, which temperature behavior, governed by the phase transition, determines mainly the observed temperature dependence of SHG in special polarization geometries. It can be completely omitted by the appropriate choice of polarization geometry. However, for general case of arbitrary geometry it also has to be taken into account. In the case of LCMO films the high value of quadrupole contribution is believed to be the result of quadrupole $\mathrm{COO}$ ordering, its quadratic behavior in temperature could be understood in terms of perturbation theory, but specification of the two discussed mechanisms (linear and quadratic in perturbation) cannot be made based on the presented experiments.

From the practical point of view quadrupole correction may be useful in order to analyze additional magneto-optical effects, which appear from higher order tensor components. As an example, deviation of the crystallographic symmetry owing to magnetization in ferro- and antiferromagnets should be possible on this basis. Another application is to investigate magnetization squared in demagnetized ferromagnets, which is useful in cases when magnetization switching is impossible.

\section{ACKNOWLEDGMENTS}

Parts of this work were supported by Verischerungsschutz (Germany), NWO (The Netherlands), Russian Ministry of Science and Education (Russia), RFBR (Grant No. 06-0217534). E.D.M. acknowledges University of Nijmegen and Universität Göttingen for hospitality. The authors thank A. Kirilyuk for fruitful discussions.

\section{APPENDIX A: AZIMUTHAL AND POLARIZATION DEPENDENCES OF THE SHG INTENSITY FOR RHOMBOHEDRAL AND CUBIC CRYSTAL}

For pure cubic symmetry $(\mathrm{m} 3 \mathrm{~m})$ the SHG field is induced by the quadrupole term (2) which for both $p-s$ and $s-s$ polarization combinations can be written as

$$
E_{2 \omega}^{Q}(\Psi) \propto\left(\chi_{x x x x}+\chi_{x x y y}-2 \chi_{x y x y}\right) \sin (4 \Psi) E^{2}(\omega),
$$

i.e., the intensity dependence contains eight equal maxima. The magnetic-dipole MDM term (3) is identical to zero, because for the $m 3 m$ symmetry group, the axial tensor $\chi_{0 i j k}^{M D M}$ contains only one independent component which must meet the condition: $\chi_{x y z}^{M D M}=-\chi_{x z y}^{M D M}$. Because from Eq. (3) it follows that $\chi_{x y z}^{M D M}=\chi_{x z y}^{M D M}$ (for a single input beam, the indices $j, k$ can be exchanged) one gets $\chi_{0 i j k}^{M D M} \equiv 0$. For the magneticdipole MDP term (4) the latter condition does not hold, but nevertheless for $s-s$ and $p-s$ polarization combinations $E_{2 \omega}^{M D P}(\Psi) \equiv 0$.
In the absence of a net magnetization, the polarization dependences of $s$-polarized SHG for the azimuthal angle $\Psi$ $=0$ contain quadrupole and MDP contributions which are both twofold symmetric in the field and thus fourfold in the SHG intensity:

$$
\begin{gathered}
E^{2 \omega, Q}(\varphi) \propto\left(\chi_{x x y y}^{Q}+\chi_{x y x y}^{Q}\right) \sin 2 \varphi, \\
E^{2 \omega, M D P}(\varphi) \propto \chi_{x y z}^{M D P} \sin 2 \varphi .
\end{gathered}
$$

For $p$-polarized SHG polarization dependences also contain quadrupole and MDP contributions both containing the constant and twofold terms:

$$
\begin{aligned}
E^{2 \omega, Q}(\varphi) \propto & 3(1+\sqrt{3})\left(\chi_{x x x x}^{Q}+\chi_{x x y y}^{Q}\right)(1+\cos 2 \varphi)+8(1 \\
& +\sqrt{3}) \chi_{x y x y}^{Q} \\
E^{2 \omega, M D P}(\varphi) \propto 4 & \chi_{x y z}^{M D P}((1+3 \sqrt{3})-3(1+\sqrt{3}) \cos 2 \varphi) .
\end{aligned}
$$

When the net magnetization is nonzero both magnetization induced terms (7) and (8) contribute to the nonlinear optical signal:

$$
\begin{gathered}
E^{2 \omega, P M I}(\varphi) \propto M\left(\chi_{x x x x}^{P M I}-\chi_{x y y x}^{P M I}\right) \sin 2 \varphi, \\
E^{2 \omega, M M I}(\varphi) \propto M\left(\left(\chi_{x x x x}^{M M I}+\chi_{x y x y}^{M M I}\right)+\left(\chi_{x x x x}^{M M I}-\chi_{x y x y}^{M M I}\right) \cos 2 \varphi\right) .
\end{gathered}
$$

A structural analysis by transmission electron microscopy shows that the symmetry of the film is rhombohedral (quasicubic) with 3- $m$ point group. For this symmetry, the situation is more complicated. The quadrupole tensor is determined for the rhombohedral symmetry $\left(C_{3}\left\|z, C_{2}\right\| y\right)$ by ten independent tensor components: $\chi_{z z z z}^{Q}, \chi_{x x y y}^{Q}, \chi_{x x x z}^{Q}, \chi_{x x x x}^{Q}$ $=2 \chi_{x x y y}^{Q}+\chi_{x y x y}^{Q}, \chi_{z x x x}^{Q}, \chi_{x x z x}^{Q}, \chi_{x y x y}^{Q}, \chi_{x x z z}^{Q}, \chi_{z x x z}^{Q}, \chi_{x z x z}^{Q}, \chi_{z x z x}^{Q}$.

For $s-s$ and $p-s$ polarization combinations, the azimuthal dependence of the SHG field produced by the quadrupole terms contains one-, two-, and threefold dependences in $\Psi$, in addition to the fourfold:

$$
\begin{aligned}
E^{2 \omega}(\Psi) \approx & c_{1} \cos \Psi+s_{1} \sin \Psi+c_{2} \cos 2 \Psi+c_{3} \cos 3 \Psi \\
& +s_{3} \sin 3 \Psi+s_{4} \sin 4 \Psi
\end{aligned}
$$

where as an example $c_{1}=2 \chi_{z z z z}+\sqrt{2} \chi_{x x z x}-2\left(2 \chi_{x x y y}+\chi_{x y x y}\right.$ $-2 \chi_{y y z z}+\chi_{y z y z}+2 \chi_{z z x x}-\chi_{z x z x}$.

For $s-s$ and $p-s$ polarization combinations, the MDM term equals zero, while the MDP term is nonzero and contains first, second, and third harmonics and does not contain the fourth harmonic in $\Psi$ :

$$
\begin{aligned}
E^{2 \omega}(\Psi) \approx & \left(\sqrt{2} \chi_{y y y}-4 \chi_{x y z}+4 \chi_{z x y}\right)(\cos \Psi+\sin \Psi)+\left(\sqrt{2} \chi_{y y y}\right. \\
& \left.-\chi_{x y z}+\chi_{z x y}\right) \cos 2 \Psi+\chi_{y y y}(\cos 3 \Psi-\sin 3 \Psi)
\end{aligned}
$$


The polarization dependences for both $p$ - and $s$-out SHG can be written as in Eq. (12) where the coefficients $F_{0}, C_{0}, S_{0}$ contain contributions (2), (4), (8), and (9), i.e., all contributions except the MDM term.

Azimuthal dependences $I_{2 \omega}(\Psi)$ were fitted based on two point symmetry groups: cubic, using Eq. (A1), and rhombohedral, using Eq. (A8). At room temperature the LCMO film is in the paramagnetic state, therefore only crystallographic contributions were taken into account. Dashed and solid lines in Fig. 3(a) and 3(b) show the fits based on $m 3 m$ and 3-m point symmetry groups, respectively. It can be seen from the figures that the deviation from the perfect eightfold symmetry is quite small. Quantitatively we can estimate these deviations from the ratios of the Fourier components for rhombohedral symmetry to the ones of the cubic symmetry:

$$
c_{1}: s_{1}: c_{2}: c_{3}: s_{3}: \Delta s_{4}: s_{4}=0.04: 0.03: 0.07: 0.01: 0.01: 0.002: 1
$$
and

$$
c_{1}: s_{1}: c_{2}: c_{3}: s_{3}: \Delta s_{4}: s_{4}=0.03: 0.01: 0.02: 0.05: 0.03: 0.001: 1
$$

for $s-s$ and for $p-s$ polarization combinations, respectively. Thus, the average deviation of the crystal symmetry from cubic in terms of nonlinear susceptibilities does not exceed $7 \%$ and from the point of view of nonlinear optics the system can be considered as cubic $(\mathrm{m} 3 \mathrm{~m})$.

The polarization dependences are also fitted for both symmetries, using Eqs. (A2)-(A5) for cubic and Eq. (12) for rhombohedral symmetry. It is not possible to distinguish between these two symmetries for a magnetized sample, but for a demagnetized (ZFC) one they are different. The best fit for a demagnetized sample was obtained for the cubic model (Fig. 4).

Thus, both the azimuthal and polarization dependences of the SHG intensity in LCMO film confirm the symmetry of the sample to be very close to cubic, and this symmetry is used in the following to analyze all other dependences. Based on the azimuthal and polarization dependences it is shown as well that for the demagnetized sample the main contribution for $s$-out polarization, which is mainly discussed in the following, is of quadrupole origin.

\section{APPENDIX B: CALCULATIONS OF MAGNETIZATION- INDUCED CORRECTIONS TO NONLINEAR SUSCEPTIBILITY FOR DIFFERENT ORDERS OF PERTURBATION}

The perturbation operator in Hamiltonian defining the interaction of conductive electron with exchange field $\vec{H}_{e}$ can be written as

$$
\hat{V}=-\vec{H}_{e} \hat{\vec{S}}=-\eta(\vec{r}) \vec{\mu} \hat{\vec{S}}
$$

where $\hat{\vec{S}}$ is conductivity electron spin operator. In particular, in the case of $s$ - $d$ interaction $\eta(\vec{r}) \propto \sum_{i} \delta\left(\vec{r}-\vec{R}_{i}\right)\left(\vec{R}_{i}\right.$ is the ion coordinate). Since the coordinate and spin spaces are independent, one can choose the spin quantization direction along the magnetization direction. In this case

$$
\hat{V}=-\eta(r) \mu \hat{S}^{(z)} \equiv \hat{D} \hat{S}^{(z)}
$$

where $\hat{D}$ is the coordinate part of perturbation operator $\hat{V}$.

The perturbation $\hat{V}$ influences the $m, n$, and $p$ energy electron states, and also the wave functions for these states in assumption of inhomogeneity of the exchange field within the unit cell. This results in admixing to the unperturbed wave function the ones of the states with the same wave vector but from different electron bands.

Microscopic expression for nonlinear susceptibility $\chi_{i j k}$ of any type can be written as ${ }^{25}$

$$
\hat{\chi}_{i j k} \propto \sum_{S_{z}} \sum_{m, n, p}\left[\frac{\left(A_{i}\right)_{m n}\left(B_{j}\right)_{n p}\left(C_{k}\right)_{p m}}{\left(2 \omega-\omega_{n m}\right)\left(\omega-\omega_{p m}\right)}+\cdots\right]
$$

where $\vec{A}, \vec{B}, \vec{C}=\vec{M}, \vec{P}, \vec{Q}$ are the MD, ED, and Q momentum operators (not containing spin operator), $\hbar \omega_{n g}$ and $\hbar \omega_{n^{\prime} g}$ are the energy differences between the ground and the excited state and between the ground and intermediate states, respectively. Since $m, n$, and $p$ relate to the same value of $S_{z}$, contributions from electrons with opposite spins are additive.

Linear in $\hat{V}$ correction to nonlinear susceptibility can be written as

$$
\hat{\chi}_{i j k}^{(1)} \propto \sum_{S_{z}} S_{z} \sum_{m, n, p, q}\left[\frac{D_{m n}\left(A_{i}\right)_{n p}\left(B_{j}\right)_{p q}\left(C_{k}\right)_{q m}}{\omega_{m n}\left(2 \omega-\omega_{q m}\right)\left(\omega-\omega_{p m}\right)}+\cdots\right],
$$

where the states $m, n, p$, and $q$ correspond to the same value of $S_{z}$.

After summation over $S_{z}$ the value of $\hat{\chi}_{i j k}^{(1)}$ (and of all corrections odd in $\hat{V}$ as well) equals zero because the second sum in (B4) does not depend on $S_{z}$ due to Kramer's degeneration.

The second order in $\hat{V}$ correction to nonlinear susceptibility can be written as

$$
\begin{aligned}
\hat{\chi}_{i j k}^{(2)} \propto & \sum_{S_{Z}} S_{z}^{2} \sum_{m, n, p, q, r}\left[\frac{D_{m n}\left(A_{i}\right)_{n p}(D)_{p q}\left(B_{j}\right)_{q r}\left(C_{k}\right)_{r m}}{\omega_{m n}\left(2 \omega-\omega_{r m}\right)\left(\omega-\omega_{p m}\right)\left(\omega-\omega_{q m}\right)}\right. \\
& +\cdots] \propto \mu^{2} .
\end{aligned}
$$

For all corrections to $\hat{\chi}_{i j k}$ even in $\hat{V}$ contributions from electrons with opposite spins $S_{z}$ are equal and do not compensate each other. 
Quadratic in $S_{z}$ but linear in magnetization correction to $\hat{\chi}_{i j k}$ can be obtained with relativistic spin-orbital interaction being taken into account with the Hamiltonian

$$
\hat{H}_{S 0}=\Im[\nabla W(\vec{r}), \hat{\vec{p}}] \hat{\vec{S}}
$$

where $\mathfrak{I}$ is the constant, $W(\vec{r})$ and $\hat{\vec{p}}$ are electron potential energy and momentum operator, respectively. This correction can be written as

$$
\begin{aligned}
\hat{\chi}_{i j k}^{(3)} \propto & \sum_{S_{Z}} S_{z}^{2} \sum_{m, n, p, q, r}\left[\frac{D_{m n}\left(A_{i}\right)_{n p}(E)_{p q}\left(B_{j}\right)_{q r}\left(C_{k}\right)_{r m}}{\omega_{m n}\left(2 \omega-\omega_{r m}\right)\left(\omega-\omega_{p m}\right)\left(\omega-\omega_{q m}\right)}\right. \\
& +\cdots] \propto \mu,
\end{aligned}
$$

where $\hat{E}=\Im\lfloor\nabla W(\vec{r}), \hat{\vec{p}}\rfloor_{z}$ is the $z$ projection of the vector product.

All corrections odd in magnetization are relativistic small, because they can be obtained only if the relativistic spinorbital interaction is taken into account.
${ }^{1}$ A. Goyal, M. Rajeswari, R. Shreekala, S. E. Lofland, S. M. Bhagat, T. Boettcher, C. Kwon, R. Ramesh, and T. Venkatesan, Appl. Phys. Lett. 71, 2535 (1997).

${ }^{2}$ J. Z. Sun, L. Krusin-Ellbaum, P. R. Duncombe, A. Gupta, and R. B. Laibowitz, Appl. Phys. Lett. 70, 1769 (1997).

${ }^{3}$ J. Nickel and S. Zhang, U.S. Patent No. 5,835,003 (10 November 1998).

${ }^{4}$ E. Dagotto, Science 309, 257 (2005).

${ }^{5}$ A. V. Petukhov, Th. Rasing, T. Katayama, N. Nakajima, and Y. Suzuki, J. Appl. Phys. 83, 6742 (1998).

${ }^{6}$ K. Postava, D. Hrabovsky, J. Pištora, A. R. Fert, Š. Višňovsky, and T. Yamaguchi, J. Appl. Phys. 91, 7293 (2002).

${ }^{7}$ Th. Rasing, J. Magn. Magn. Mater. 175, 35 (1997).

${ }^{8}$ V. N. Gridnev, V. V. Pavlov, R. V. Pisarev, A. Kirilyuk, and Th. Rasing, Phys. Rev. B 63, 184407 (2001).

${ }^{9}$ M. Fiebig, D. Frцhlich, G. Sluyterman v. L., and R. V. Pisarev, Appl. Phys. Lett. 66, 2906 (1995).

${ }^{10}$ V. Kirilyuk, A. Kirilyuk, and Th. Rasing, Appl. Phys. Lett. 70, 2306 (1997).

${ }^{11}$ V. Moshnyaga, I. Khoroshun, A. Sidorenko, P. Petrenko, A. Weidinger, M. Zeitler, B. Rauschenbach, R. Tidecks, and K. Samwer, Appl. Phys. Lett. 74, 2842 (1999).

${ }^{12}$ V. Moshnyaga, L. Sudheendra, O. I. Lebedev, S. A. Köster, K. Gehrke, O. Shapoval, A. Belenchuk, B. Damaschke, G. van Ten- deloo, and K. Samwer, Phys. Rev. Lett. 97, 107205 (2006).

${ }^{13}$ Ch. Hartinger, F. Mayr, A. Loidl, and T. Kopp, Phys. Rev. B 70, 134415 (2004).

${ }^{14}$ V. Moshnyaga, B. Damaschke, R. Tidecks, and K. Samwer, J. Appl. Phys. 93, 6354 (2003).

${ }^{15}$ M. Fiebig, V. V. Pavlov, and R. V. Pisarev, J. Opt. Soc. Am. B 22, 96 (2005)

${ }^{16}$ R. R. Birss, Symmetry and Magnetism (North-Holland, Amsterdam, 1966).

${ }^{17}$ J. E. Sipe, D. J. Moss, and H. M. van Driel, Phys. Rev. B 35, 1129 (1987).

${ }^{18}$ W. N. Herman and L. M. Hayden, J. Opt. Soc. Am. B 12, 416 (1995).

${ }^{19}$ D. Kim, B. Revaz, B. L. Zink, F. Hellman, J. J. Rhyne, and J. F. Mitchell, Phys. Rev. Lett. 89, 227202 (2002).

${ }^{20}$ D. P. Arovas and F. Guinea, Phys. Rev. B 58, 9150 (1998).

${ }^{21}$ R. Osborn, S. Rosenkranz, D. N. Argyriou, L. Vasiliu-Doloc, J. W. Lynn, S. K. Sinha, J. F. Mitchell, K. E. Gray, and S. D. Bader, Phys. Rev. Lett. 81, 3964 (1998).

${ }^{22}$ D. I. Khomskii and K. I. Kugel, Phys. Rev. B 67, 134401 (2003).

${ }^{23}$ L. Sudheendra et al., cond-mat/0605712 (unpublished).

${ }^{24}$ G. Lefkidis and W. Hübner, Phys. Rev. Lett. 95, 077401 (2005).

${ }^{25}$ Y. R. Shen, The Principles of Nonlinear Optics (Wiley, 2003). 